\title{
Process and Goal \\ in White Hmong
}

\author{
Nerida Jarkey
}

\section{Introduction}

My investigation of the White Hmong language was made possible through the kindness and generosity of my primary language teacher, Cua Lis, and her family: Cua’s husband Choj (Sao), Choj's brothers Yeeb, Nkaj Yias (Gary), and Ntxawg (Yeu), and their dear mother Maiv Yaj. I also received enthusiastic help from Charlie Sayaxang, Sourivan Thongpao, Maiv Dub Yaj, and Ge and Niaj Pov Lis. Cua and her family originally came from Xieng Khoung province in Laos and, at the time I conducted the basic research for this investigation during the mid 1980s to early 1990s, they lived in Sydney, Australia.

To all of these wonderful people, and especially to Cua, I am grateful not only for help in learning about language but also for help in learning about life. Quite early on in the course of my research I gave birth to my first child, Alex. Cua already had her first son, William, and had recently given birth to her second, Matthew. Cua was like a big sister to me and I treasure, more than I can say, her friendship and guidance in those early years of motherhood. Not too far along the track, my second son, Dom, 
and Cua's daughter, Melanie, arrived too. Perhaps it's not surprising that my memories of visiting Cua to learn Hmong are intricately tied with my memories of pregnancy, birth, exhaustion, joy, and lots and lots of noise!

As we sat feeding our babies together, a casual observer might have been struck by our differences: Cua short, darkskinned, and glowing with good health and strength, and I, tall, fair-skinned and looking (and feeling) as if a slight breeze would blow me over. Yet one warm and reassuring thing that Cua often used to say as we sat down together to feed the babies still stands out in my memory: 'We're all the same'!

In some ways, that's just what this chapter is all about. In it I discuss an aspect of language - the expression of process and goal - in which Hmong and English appear to exhibit very different modes of expression. While these differences are extremely interesting and important to acknowledge, I conclude, however, that they should not be taken as indicative of any underlying differences in the way that speakers of these two languages fundamentally conceive of the notions described. They should simply be understood, instead, as attributable to the way that two typologically distinct languages distribute semantic information within the clause.

\section{Summary of the Argument}

This chapter investigates how processes that have goals are represented in the White Hmong language, and discusses the ways in which the representation of such processes in Hmong differs from that in English. The chapter deals with two kinds of processes: those that have intrinsic goals and those that have extrinsic goals.

Processes that have intrinsic goals, like xaws ib daig tiab 'sew a skirt', are telic: they are processes that come to an inevitable terminal point, after which they can no longer continue. In other words, if you keep on 'sewing a skirt', you will eventually 'have sewn the skirt'; you just can't keep on sewing the same skirt forever! This kind of process is quite different from a process that has an 
extrinsic goal, such as nrhiav kuv nti nplhaib 'search for my ring'. Processes like this are atelic: they have no inevitable terminal point. One might keep on 'searching for one's ring' forever, without ever finding it.

The primary difference between English and Hmong with respect to these goal-oriented processes is related to the way in which the attainment of the goal is expressed. In English, the attainment of goal of the two different kinds of goal-oriented processes - those with intrinsic goals and those with extrinsic goals - is expressed in two quite different ways. To express the attainment of an intrinsic goal one simply uses a different form of the same verb that is used to describe the goal-oriented process, while to express the attainment of an extrinsic goal one must use an entirely different verb.

In Hmong, however, the attainment of both an intrinsic and an extrinsic goal is expressed in basically the same way. When the focus is on the process itself, a simple verb is used. When the focus is on the attainment of the goal (whether intrinsic or extrinsic), a Serial Verb Construction is used. In these 'Attainment Serial Verb Constructions', two verbs appear in a single clause: the first describes the process and the second describes the attainment of the goal.

These differences in expression should not be thought of as suggesting any fundamental difference between the ways in which speakers of these two languages conceptualise the notion of attainment of goal. Instead, it should simply be seen as a result of the way in which these two typologically distinct languages deal with this particular language function.

\section{A First Glance at the Differences: Hmong and English}

There are a number of different kinds of actions involving processes that have goals. Observe the following examples in Hmong and their translations in English: 


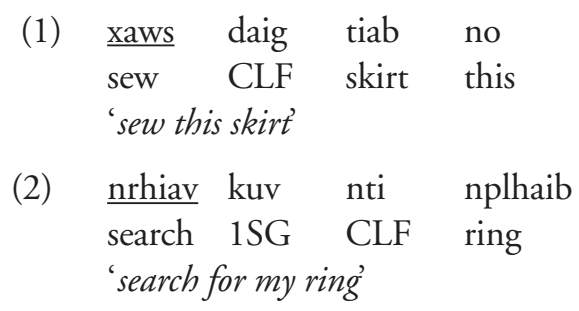

Both of these examples describe an action that occurs over time (a process), and that has a clear purpose (a goal). One sews a skirt (Example (1)) in order to get a new skirt. One searches for a ring (Example (2)) in order to find the ring.

There are some interesting differences, however, in the ways in which the attainment of the goals of these two processes is expressed in Hmong and in English. These are shown in Examples (1') and (2').

(1')

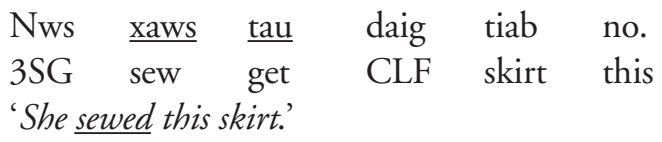

(2') Kuv nrhiav tau
1SG search get

The Hmong sentences in (1') and (2') are similar to one another, and also similar to the Hmong examples (1) and (2) respectively. In each case, the attainment of the goal is expressed by simply adding the verb tau 'get' after the verb that indicates the goal-oriented process ( $\underline{\text { xaws }}$ 'sew' in (1') and nrhiav 'search' in (2')). Looking only at the Hmong examples (1) and (1'), we might assume that tau is simply a marker of past tense: $\underline{x a w s}$ 'sew', $\underline{\text { xaws }}$ tau 'sewed'. Examples (2) and (2') show us clearly that this is not the case. While nrhiav in (1) means 'search', nhriav tau in (1') means not 'searched' but 'found'. The verb tau 'get' appearing after the verbs xaws and nhriav in these sentences functions not to mark past tense, but to indicate that the goal is attained. ${ }^{1}$ Sequences of two verbs such as these, juxtaposed within a single clause with no conjunction or complementizer, are known as Serial Verb Constructions. I will refer to the type of construction illustrated above in Hmong as the 'Attainment Serial Verb Construction'. 
The way in which the attainment of goal is expressed in the English version of (1') is different in one respect from the Hmong example, but similar to it in another. While English does not introduce a second verb, as in the Hmong counterpart with tau 'get', it does utilise the same verb, simply in a different form -

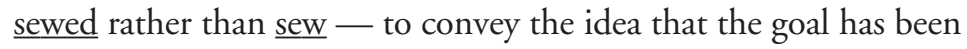
attained.

In the English version of (2'), on the other hand, the attainment of the goal is expressed in a totally different way. Rather than repeating, in a different form, the same verb ( $\underline{\text { search }}$ ) that is used to describe the goal-oriented process, an entirely different verb (ind) is introduced to describe the attainment of the goal. ${ }^{2}$

\section{Types of Process: Activities and Accomplishments}

In order to understand more about these very different ways of expressing attainment of goal in these two languages, it will be helpful to introduce a well-known distinction between types of processes: the distinction between atelic and telic processes: 'Activities' and 'Accomplishments' (Vendler 1967, Dowty 1979). Here are some examples of each of these process types.

(3) 'Activities' (atelic): laugh, run, watch TV, drive a car, search for a ring

'Accomplishments' (telic): run a mile, write a letter, mould a rice cake, sew a skirt

Activities, such as 'laugh', 'run', 'watch TV', 'drive a car', and so on, describe processes that occur over a period of time and which do not involve any change or result occurring as an inherent part of that process. Theoretically one could go on 'running' or 'driving a car' for any length of time. Although extrinsic factors, such as exhaustion or the end of the road, might cause one to 'stop' at some point, there is nothing intrinsic to the actions of 'running' or 'driving a car' themselves that leads to them inevitably being 'finished'. 
Accomplishments, such as 'run a mile', 'write a letter', or 'mould a rice cake', on the other hand, have both a process component and a result component. If you persevere for long enough with the process of 'writing a letter' or 'moulding a rice cake', you will ultimately achieve the result of having 'written a letter' or 'moulded the rice cake'. Because they involve these two components - a process and its inherent result — Accomplishments have sometimes been characterised as a kind of amalgam of an Activity and a result (for example, Jacobsen 1992: 162). ${ }^{3}$

Although Accomplishments are quite often characterised in this way — as an Activity that leads up to a result - it is important to recognise that the process component of an Activity differs from that of an Accomplishment in a fundamental way. While an Activity involves a homogeneous process - one that is basically the same at its beginning and its end - an Accomplishment involves a non-homogeneous process - one that moves towards the attainment of its own, inherent goal.

The difference between these two types of event is not, however, as this may seem to imply, that Accomplishments have a goal and Activities do not. Instead, Accomplishments differ from Activities in that they have an intrinsic goal. A goal of this kind is often appropriately referred to as a 'result' or a 'terminal point'. Accomplishments are 'telic': they are 'directed toward attaining a goal or limit at which the action exhausts itself and passes into something else' (Andersson 1972). ${ }^{4}$ In other words, you just can't keep on 'moulding the same rice cake' or 'sewing the same skirt' forever.

Activities may or may not have a goal. You do not have a goal when you 'laugh', for example, but you do when you 'search for your ring'. If Activities do have a goal, however, it must be an extrinsic one, because Activities are 'atelic'. Take the Activites 'search for your ring' and 'chase the kids', for example, both of which have clear, but extrinsic, goals:
a. search for your ring
b. chase the kids

You can persevere for an indefinite period of time 'searching for your ring' or 'chasing the kids' but in the end you may have 
done no more than that; you may never actually attain your goal of 'finding your ring' or 'catching up with kids'. A goal of this kind is something quite separate from the action described, rather than being a natural 'result' or 'terminal point' of that action. It thus differs in a fundamental way from the intrinsic, telic goal of an Accomplishment.

\section{Attainment of Goal in Activities and Accomplishments}

Returning, then, to our original discussion concerning the ways in which the attainment of the goal of a process is expressed in Hmong and in English, we can now use this distinction between Activities and Accomplishments to characterise the differences we have observed.

In English, the attainment of the intrinsic goal of an Accomplishment, such as sew a skirt, is expressed by simply using the same predicate in a different form. Either a past form (sewed a skirt) or a perfect form (has sewn a skirt) will suffice to convey that the process of 'sewing a skirt' has been carried through to its natural terminal point, the creation of a new skirt.

The attainment of the extrinsic goal of a goal-oriented Activity, such as search for a ring, on the other hand, cannot possibly be expressed by simply using the same predicate in English. Neither the past form (searched for a ring) nor the perfect form (has searched for a ring) can serve to convey that the goal has been attained, because the attainment of that goal is not an intrinsic part of the meaning of the predicate. In order to convey the attainment of the goal, it is necessary to employ an entirely different verb (find) in English.

In Hmong, the difference between the intrinsic goal of an Accomplishment and the extrinsic goal of a goal-oriented Activity does not result in a difference in the way that the attainment of these goals is expressed. Both the goal of the Accomplishment xaws daim tiab no 'sew this skirt' and that of the Activity nrhiav kuv nti plhaib 'search for my ring', is expressed as attained by the use of a 
Serial Verb Construction, involving the addition of a second verb after the first one: the verb tau 'get' (see examples (1') and (2')).

It is important to note that, although the verb tau 'get' is by far the most common result verb used in series with another verb to express the attainment of a goal in Hmong, it is by no means the only one. A variety of verbs can appear in this role, depending on the nature of the goal that is attained. This is illustrated in Examples (6) and (7).

(6) a. Nws mus raws lawv.

3SG go pursue 3PL

'She chased them.'

b. Nws mus raws cuag lawv.

3SG go pursue reach 3PL

'She caught up with them.'
a. Lawv mus tom khw lawm.
3PL go over.there market PERF
'They have gone off to market.'

b. Law mus txog tom khw lawm. 3PL go arrive over.there market PERF

'They have arrived at the market.'

In Example (6), the verb raws 'pursue', in the goal-oriented Activity predicate raws lawv 'pursue them', is followed by the result verb cuag 'reach' to indicate that the extrinsic goal of this Activity is attained. In (7), the verb mus 'go', in the Accomplishment predicate mus tom khw 'go to market' is followed by the result verb txog 'arrive' to indicate that the intrinsic goal of this Accomplishment is attained.

\section{More about Accomplishments in Hmong}

A question that naturally arises, when we look at Hmong examples involving Accomplishment predicates, is: 'why do we need the second verb at all to express attainment of the goal?' Surely, if a goal-oriented process is telic, and as such is 'directed toward attaining a goal or limit at which the action exhausts itself and 
passes into something else' (Andersson 1972), then simply expressing that process in the past or perfect form will be sufficient to indicate that the goal has been attained.

There seems, however, to be a subtle difference between Accomplishment predicates in Hmong and their equivalents in English. A predicate like xaws daig tiab no 'sew this skirt' or puab ib lub ncuav 'mould a rice cake' seems to involve a greater degree of focus on the process component of the event than its English counterpart, and less focus on the inherent end point.

Thus, in Hmong it is very natural to say, for example:

(8) Nws xaws daig tiab no lawm, tseem tsis tau tag.

3SG sew CLF skirt this PERF still NEG PFCV finish

*'She has sewn this skirt, (but) hasn't finished it yet.'

(9) Kuv puab ib lub ncuav lawm, tseem tsis tau tag.

1SG mould one CLF skirt PERF still NEG PFCV finish

*'I have moulded a rice cake, (but) haven't finished it yet.'

The English translations in the examples above are marked with an asterisk, because they sound so odd. When one hears 'She has sewn this skirt' in English, one would take it to mean 'She has finished sewing this skirt'. Likewise, 'I have moulded a rice cake' indicates 'I have finished moulding the rice cake'. The Hmong sentences, on the other hand, are not odd or contradictory in any way, even with perfect or past tense reference. This is because the focus of an Accomplishment predicate in Hmong, like xaws daig tiab no 'sew this skirt', is on the process leading up to the goal, rather than on the goal itself. ${ }^{5}$ Dahl (1981: 81ff) notes that Slavic aspectologists observe not one but two distinctions in relation to telicity. He gives the examples listed in (10) to illustrate:

(10) a. I was writing.

b. I was writing a letter. ('T property')

c. I wrote a letter (taken to imply 'I finished it'). ('T property' \& 'P property')

The first distinction is between predicates that do not lead up to a terminal point ((10a) above) and those that do ((10b) and (10c)). Dahl coins the term 'T property' to refer to those predicates that do lead up to a terminal point $((10 b)$ and $(10 c))$. The second 
distinction is between predicates that simply lead up to a terminal point $((10 \mathrm{~b}))$, and those for which the terminal point is actually reached $((10 \mathrm{c}))$. This latter property Dahl terms the 'P property' $((10 c))$.

It seems from examples like (8) and (9) above that Accomplishment predicates in Hmong have the 'T property' but not necessarily the 'P property'. That is, even when they occur in the past or perfect form (as in Example (8) xaws daig tiab no lawm 'sew CLF skirt this PERFECT'), they express an action that leads up to a terminal point, but for which the terminal point has not necessarily been reached. It is interesting to note that Accomplishment predicates in some other Asian-area languages, for example Mandarin and Japanese, exhibit the same kind of properties with regard to telicity (Smith 1990, Jarkey, 1998).

Because these predicates describe actions that lead up to an intrinsic goal (the 'T property'), they are, in the absence of any explicit denial of the achievement of that goal, often taken to imply that the goal is, in fact, attained. Observe the following examples:

(11) Nws xaws daig tiab no rau kuv lawm. 3SG sew CLF skirt this for 1SG PERF

'She has sewn this skirt for me.'

$\begin{array}{llllll}\text { Thaum yus puab } & \text { ib } & \text { lub } & \text { ncuav... } \\ \text { when } & \text { INDEF mould } & \text { one } & \text { CLF } & \text { rice.cake }\end{array}$

'When one moulds a rice cake...'

(13) Niaj hnub cov me nyuam mus nram pas dej. every day CLF.PL child go down pond 'Every day the children go down to the pond.'

In all three sentences above an Accomplishment predicate is used without any 'support' from an Attainment Serial Verb Construction, and yet there is a clear implication that the goal in each case is attained. ${ }^{6}$

As Smith (1990: 323) points out with regard to similar predicates in Mandarin, however, this implication of the attainment of the goal is simply part of the pragmatic interpretation of predicates like these in many contexts. 'Because 
telic events involve completion, they may be used to implicate completion.' However, the fact that this implication can be denied or negated in other contexts, shown earlier in Examples (8) and (9), indicates that completion is not part of the semantic content of the predicates themselves. Following Comrie (1976: 112), we can say that these predicates are unmarked for the feature of attainment of goal, meaning that they 'simply [say] nothing about its presence or absence'.

The fact that an Accomplishment predicate in Hmong like xaws ib daig tiab 'sew a skirt', by itself, has the 'T Property' but not necessarily the 'P Property' means, then, that it can be used with an implication that the goal is attained. Nevertheless, when these predicates are used, the focus of attention is not on the terminal point at all, but rather on the goal-oriented process. An Attainment Serial Verb Construction xaws tau (ib daig tiab) 'make (a skirt)', on the other hand, unambiguously exhibits the 'P Property'. This means that, although the process leading up to the terminal point is referred to (by the first verb), the focus in this construction is very clearly on the fact that the terminal point (indicated by the second verb) is reached.

This difference in focus is responsible for the tendency for Accomplishment predicates in Hmong to be expressed with a single verb when reporting actions, and with a Serial Construction when reporting results. Compare examples (14) a. and b.

a. Xyoo no nws ua ob daig teb.
year this 3SG do two CLF field
'This year he's working two fields.'

b. Xyoo no nws ua tau ob txhab nplej. year this 3SG do get two platform rice 'This year he grew two platforms(-worth) of rice.'

In (14a), the simple predicate ua ob daig teb (do two fields) appears in a context in which the focus of interest is on the activity of the farmer: 'This year he's working two fields'. In (14b), however, the use of the Attainment Serial Verb Construction ua tau ob nthab nplej (do get two CLF rice) is clearly appropriate. In this case, the focus is not on the activity of the farmer throughout 
the year, but on the result of that activity at the end of the year: on the product of his or her labour.

Because the Attainment Serial Verb Construction focuses explicitly on the attainment of goal, it would be completely nonsensical and contradictory to follow these sentences with a denial like the ones in Examples (8) and (9) earlier.

(15) *Nws xaws tau daig tiab no lawm, tseem tsis tau tag.

3SG sew get CLF skirt this PERF still NEG get finish

*'She has sewn this skirt, (but) hasn't finished it yet.'

\section{What Might These Differences Mean?}

The discussion above has revealed that, when it comes to the expression of attainment of goal, English requires just one verb, while Hmong prefers two. This generalisation applies not only when the predicate involves an intrinsic goal (telic, Accomplishment predicates such as xaws ib daig tiab 'sew a skirt'), but also when it involves and extrinsic goal (atelic, Activity predicates such as nrhiav kuv nti nplhaib 'search for my ring').

The discussion has also revealed that there is a subtle difference between an Accomplishment predicate in English and its nearest equivalent in Hmong. While the past or perfect form of an Accomplishment predicate in English is automatically interpreted as expressing the attainment of its inherent goal (' $\mathrm{P}$ Property'), this is not necessarily the case in Hmong. An Accomplishment predicate in Hmong needs the support of a second verb (in an Attainment Serial Verb Construction) in order to unambiguously express attainment of goal.

Differences such as these between two languages might be taken to suggest differences between the ways in which speakers of those languages conceive of the notion of 'attainment of goal'. The fact that simple Accomplishment predicates are unmarked for the feature of attainment of goal in another Asian-area languages Japanese - has led some linguists to propose this as evidence for a difference in world-view between speakers of this language and speakers of English.? 
The fact that Accomplishment predicates are unmarked for the feature of attainment of goal in Japanese, Hmong and some other Asian languages does not suggest to me that speakers of these languages do not perceive such a goal in basically the same way as speakers of English do: as a specific and unambiguously attainable point. Nor does this seem to me to constitute good evidence for the notion that speakers of these languages view the world in any way differently from speakers of English.

What this lack of markedness in Accomplishment predicates does suggest to me is that this feature is not part of the semantic content of the simple predicates concerned. It is the entire clause and not just the simple verb or predicate, of course, that is the domain of the proposition. In these languages, something beyond a simple predicate is required to express a proposition involving this particular semantic concept.

If we look beyond the simple predicate but yet within the clause in Hmong (and in other Asian-area languages such as Japanese and Mandarin) it is immediately apparent that the expression of telic events in which the goal is unambiguously attained — the expression of telic events with the 'P property' in Dahl's terms — is indeed thoroughly alive and well. As shown above, one way in which this is achieved in Hmong is through the use of a Serial Verb Construction involving the support of a result verb appearing in the same clause. Likewise, in other languages of the area, a range of highly productive and frequently occurring multi-verb constructions are prominent among strategies that are used for unambiguously expressing the attainment of the goal of a telic action (Jarkey 1998).

It is important to note that multi-verb constructions in all of these languages have other important functions beyond the aspectual function that is the focus of this chapter. Serial Verb Constructions are used in Hmong to express direction and deixis, cause and effect, and so on (see Jarkey 1991). Furthermore, the Hmong Serial Verb Construction described in this chapter is used not only to express attainment of goal, but also to express ability (Jarkey 1991: 227-31).

Regardless of its other functions, however, this construction is used extremely commonly with the aspectual function described 
above: to assert the attainment of both the intrinsic goal expressed by an Accomplishment predicate and the extrinsic goal of a goaloriented Activity predicate. These multi-verb constructions in Hmong express both a goal-oriented process and the attainment of its goal, not within a simple predicate, but nevertheless within a single clause, and thus within a single proposition.

\section{Conclusion}

The data presented in this chapter do not support the idea that speakers of Hmong might view the goal of a process in any way differently from speakers of English. While this notion is, indeed, not expressed in a simple predicate with a single verb in Hmong, it is expressed through other means - notably through the use of the Serial Verb Construction described in this chapter.

The clause, rather than the simple predicate, is the domain for the expression of the proposition. If we find incongruence at the level of the predicate between English and Hmong, this may not itself constitute good evidence for assuming differences in cultural perceptions and world-views between speakers of these two languages. It may, instead, simply be evidence for differences in the ways in which the semantic load is distributed in the clause in languages with very different typological characteristics. In this case, at least, we can confidently affirm the sentiments of my friend and teacher Cua: at first glance we may look very different but, underneath, we're all the same! 


\section{Footnotes}

${ }^{1}$ A different, though perhaps related morpheme, tau, occurs preverbally in White Hmong, and is even more likely to be assumed to signal past tense. The function of this morpheme is actually to mark perfective aspect - to indicate that an event is completed, or will be completed, at the time referred to (see Jarkey 1991: 76-7). In fact there is no morpheme in Hmong that has the simple function of marking past tense.

${ }^{2}$ There are some sets of minimal pairs in English, in which a verb with a prepositional object indicates a goal-oriented action, and the corresponding verb with a direct object indicates the related result: for example, 'shoot at a monkey' versus 'shoot a monkey'; 'kick at someone' versus 'kick someone'. In Swedish, the distinction is more systematic (Dahl 1981:87), for example, stickade på en tröya 'knitted at a jumper' versus stickade en tröya 'knitted a jumper'. In some non-Germanic European languages (for example, Russian, Ancient Greek), a similar distinction is made morphologically, by employing different aspectual forms of the same verb.

3 Jacobsen uses Vendler's terminology 'Achievement' (Vendler 1967) rather than the word 'result'. Jacobsen attributes this characterisation of Accomplishments to personal communication from James McCawley (Jacobsen 1992: 253, fn 61).

${ }^{4}$ This translation from the original German is from Dahl (1981).

${ }^{5}$ Perhaps better translations might be: 'She has been sewing this skirt ...' and 'I have been moulding this rice cake'. It is important to note, however, that there is no explicit marker of imperfective aspect in these Hmong sentences. Imperfective aspect is overtly indicated in White Hmong either by reduplication of the verb, or by the use of an adverb such as tab tom 'have just begun to', tseem 'still', pheej 'continually', sij 'repeatedly', and so on (Jarkey 1991: 75).

${ }^{6}$ The types of contexts in which this implication tends to be reinforced share the feature of 'boundedness' (Dahl 1981, Hopper and Thompson 1980: 285-6).

${ }^{7}$ The highly respected Japanese linguist, Yoshihiko Ikegami, for example, argues that this supports his contention that the English language has a tendency toward a precise, 'individuum' schema, and the Japanese language toward a vague, 'continuum' schema (Ikegami 1981, 1993). He suggests that Japanese tends to be 'oriented toward blurred articulation and a focus on the whole configuration or gestalt and on the continuum' (1993: 813) as opposed to the clearer articulation and focus on the 'individuum' of English. 